\title{
Nondecoupling of Heavy Fermions and a Special Yukawa Texture
}

\author{
M. N. REBELO* \\ CERN, Department of Physics, Theory Division \\ CH-1211 Genève 23, Switzerland \\ margarida.rebelo@cern.ch
}

October 31, 2018

\begin{abstract}
Talk based on work entitled "Yukawa textures, new physics and nondecoupling," done in collaboration with G. C. Branco and J. I. SilvaMarcos, arXiv:hep-ph/0612252, to appear in Phys. Rev. D. In this work we pointed out that New Physics can play an important rôle in rescuing some of the Yukawa texture zero ansätze which would otherwise be eliminated by the recent, more precise measurements of $V_{C K M}$. We have shown that the presence of an isosinglet vector-like quark which mixes with standard quarks, can render viable a particularly interesting four texture zero Yukawa ansatz. The crucial point is the nondecoupling of the effects of the isosinglet quark, even for arbitrary large values of its mass.
\end{abstract}

\section{Introduction}

There have been many attempts at understanding the pattern of quark and lepton masses as well as mixing, including the addition of family symmetries and the introduction of special textures for the Yukawa couplings. The

\footnotetext{
* On sabbatical leave from Departamento de Física and Centro de Física Teórica de Partículas (CFTP), Instituto Superior Técnico, Av. Rovisco Pais, 1049-001 Lisboa, Portugal. E-mail: rebelo@ist.utl.pt
} 
underlying idea is that such models could be derived from grand unified models, or possibly models with extra dimensions, and be related to special symmetries.

The recent experimental discovery of neutrino oscillations pointing towards non-vanishing neutrino masses, has rendered the flavour puzzle even more exciting. Nonzero neutrino masses are evidence of physics beyond the Standard Model and have profound implications in the physics of the leptonic sector [1]. Indeed this opens the possibility of having leptonic CP violation both at low and high energies.

The dynamical generation of the Baryon Asymmetry of the Universe (BAU) is also a fundamental challenge both for particle physics and cosmology. It has been established that CP violation present in the Standard Model (SM) through the Kobayashi-Mashawa mechanism, is not sufficient to generate the observed BAU [2]. As a result, the need for a viable baryogenesis provides the strongest argument in favour of the existence of new sources of CP violation. Leptogenesis [3] is one of the most elegant and plausible scenarios for generation of BAU, specially due to the recent experimental evidence for a non-vanishing mass for neutrinos.

The availability of increasingly more precise knowledge of the mixing matrices both in the quark and lepton sectors constitutes a great challenge to flavour models.

At present all experimental data on flavour physics and $\mathrm{CP}$ violation in the quark sector are in agreement with the SM and its KM mechanism. This is a remarkable success, since it is achieved with a relatively small number of parameters. Once the experimental values of $\left|V_{u s}\right|,\left|V_{c b}\right|$ and $\left|V_{u b}\right|$ are used to fix the angles $\theta_{12}, \theta_{23}$ and $\theta_{13}$ of the standard parametrization, one has to fit with a single parameter $\delta_{13}$, the experimental values of $\epsilon_{K}, \sin (2 \beta), \Delta M_{B_{d}}$, as well as $\Delta M_{B_{s}}$ and $\alpha$.

Recently a precise measurement of the angle $\beta$ [4] [5] [6] [7] has been achieved together with the recent measurement of the rephasing invariant angles $\gamma$ [8] [9] [10] [11] [12] and $\alpha$ [13], [14] [15] [16] and the measurement of $B_{s}^{0}-\bar{B}_{s}^{0}$ mixing [17] [18. These recent measurements will be improved in the future. The angle $\gamma$ plays a crucial rôle in providing irrefutable evidence for a complex CKM matrix [19] all these measurements place constraints on the size of New Physics contributions [20] [21] [22] [23] [24] [25].

Furthermore the BaBar and Belle experiments have recently presented evidence for $D^{0}-\bar{D}^{0}$ mixing [26] [27] with no hint for new physics [28], at least not yet [29] 30] 31. 
In spite of the impressive results on flavour physics and $\mathrm{CP}$ violation in the quark sector there is still plenty of room for the presence of New Physics. Furthermore it has been observed [32] that the size of $\left|V_{u b}\right|$ is somewhat above the range favoured by the measurement of $\sin (\beta)$. Another small discrepancy is the fact that the central value of the SM prediction [33] [34] for the inclusive radiative $\bar{B} \rightarrow X_{s} \gamma$ decay, with $\bar{B}=\bar{B}^{0}$ or $B^{-}$, is now more than $1 \sigma$ [35] below the experimental average [36] [37] 38] 39].

In what follows we describe how the presence of an isosinglet vector-like quark which mixes with the standard quarks can render viable a particularly interesting four texture zero ansatz which has been recently ruled out by experiment [40].

Isosinglet vector-like quarks play a rôle very similar to righthanded singlet neutrinos in the leptonic sector in the seesaw framework [41] 42, [43] [44] 45]. Models with vectorial isosinglet quarks together with righthanded singlet neutrinos and an extra complex singlet Higgs field allow for a common origin for all CP violations [46] [47] [48] including the possibility of leptogenesis. Furthermore, these models provide a possible solution to the strong CP problem [49] [50] of the type proposed by Nelson [51] [52] and Barr [53] as was previously shown [54]. Isosinglet quarks give rise to deviations from unitarity of the $V_{C K M}$ matrix and $\mathrm{Z}$ flavour changing neutral currents [55] [56] 57] [58] [59] 60] producing new physics effects which may be observed in the LHC in the near future [61].

\section{Four Zero Hermitian Ansatz embedded into a Model with One Down Vectorial Quark}

In our work we considered a specially interesting four zero Hermitian ansatz which had been analysed in detail in the literature [62] 63] 64] where the quark mass matrices $M_{u}, M_{d}$ are assumed to have the form:

$$
M_{u}=\lambda_{u} \quad K_{u}^{\dagger}\left[\begin{array}{ccc}
0 & a_{u} & 0 \\
a_{u} & b_{u} & c_{u} \\
0 & c_{u} & 1-b_{u}
\end{array}\right] K_{u} \quad ; \quad M_{d}=\lambda_{d} \quad\left[\begin{array}{ccc}
0 & a_{d} & 0 \\
a_{d} & b_{d} & c_{d} \\
0 & c_{d} & 1-b_{d}
\end{array}\right]
$$

with $K_{u}=\operatorname{diag}\left(e^{i \phi_{1}}, 1, e^{i \phi_{3}}\right)$ and all other parameters real.

The Hermitian matrices given by Eq. (11) give rise to leading order to the 
well known texture zero relations 65] 66] 67] 64]:

$$
\left|\frac{V_{u b}}{V_{c b}}\right|=\sqrt{\frac{m_{u}}{m_{c}}} \quad\left|\frac{V_{t d}}{V_{t s}}\right|=\sqrt{\frac{m_{d}}{m_{s}}} \quad\left|V_{u s}\right|=\left|\sqrt{\frac{m_{d}}{m_{s}}} e^{i \phi_{1}}-\sqrt{\frac{m_{u}}{m_{c}}}\right|
$$

The requirement of Hermiticity is important in order to render texture zero ansätze predictive, it was shown [68] that without this requirement several interesting zero textures would simply correspond to a choice of weak basis.

It was already pointed out before [64] that the relation obtained for $\left|V_{u b}\right| /\left|V_{c b}\right|$ strongly disfavoured this ansatz due to the smallness of the ratio $\sqrt{\frac{m_{u}}{m_{c}}}$. In the meantime the experimental value [32] for $\left|V_{u b}\right|$ went up significantly and this constraint became even more stringent. The determination of the ratio $\left|V_{t d}\right| /\left|V_{t s}\right|$ has been significantly improved and is theoretically clean, its present value [32] lies below the ratio $\sqrt{\frac{m_{d}}{m_{s}}}$ therefore also disfavouring this ansatz. Another source of difficulties lies in $\sin 2 \beta$ since ansätze such like the one we are considering have no non-factorizable phases and therefore produce too small values [69] for the angle $\beta$. Furthermore in the framework of this ansatz the value of $\gamma$ is very constrained and tends towards too large a value which may be ruled out once the experimental errors are reduced.

The difficulty in accommodating $\beta$ and the ratio $\left|V_{t d}\right| /\left|V_{t s}\right|$ could be avoided by assuming that there are new physics contributions to $B_{d}^{0}-\bar{B}_{d}^{0}$ and $B_{s}^{0}-\bar{B}_{s}^{0}$ mixings. However the remaining difficulties with the extraction of $\left|V_{u b}\right| /\left|V_{c b}\right|$ and $\gamma$ would not be solved since they are unaffected by the presence of new physics in the mixing.

In order to overcome these problems we embedded the above ansatz into an extension of the SM with one additional $Q=-1 / 3$ isosinglet vectorlike quark. Therefore we analysed the larger ansatz obtained with the same choice for $M_{u}$ and $M_{d}$ placed in the new $4 \times 4$ down quark matrix $\mathcal{M}_{d}$ in the following way:

$$
\mathcal{M}_{d}=\left(\begin{array}{cc|c} 
& & 0 \\
M_{d} & & 0 \\
- & & 0 \\
M_{D} & & -
\end{array}\right)
$$

With only one extra vectorial quark $M_{D}$ is a $1 \times 3$ matrix and $H$ a single entry. Since the mass terms $M_{D}, H$ are $S U(2) \times U(1)$ invariant they can be much larger than the electroweak scale. We may assume that there is a family symmetry which leads to the four texture zero ansatz, in the $3 \times 3$ 
quark mass matrices involving standard quarks which is softly broken by the terms $M_{D}$ and $H$. The matrix $\mathcal{M}_{d}$ is diagonalized by the usual bi-unitary transformation:

$$
U_{L}^{\dagger} \mathcal{M}_{d} U_{R}=\left(\begin{array}{cc}
\bar{m} & 0 \\
0 & \bar{M}
\end{array}\right)
$$

where $\bar{m}=\operatorname{diag}\left(m_{d}, m_{s}, m_{b}\right)$ and $\bar{M}$ is the heavy quark mass. One can write $U_{L}$ in block form,

$$
U_{L}=\left(\begin{array}{cc}
K & R \\
S & T
\end{array}\right)
$$

where $K$ is the usual $3 \times 3 V_{C K M}$ matrix. $U_{L}$ is the matrix that diagonalizes $\mathcal{M}_{d} \mathcal{M}_{d}^{\dagger}$, and the following relations can be readily derived [54] in the limit $M_{D}, H>>\mathcal{O}\left(M_{d}\right)$

$$
\begin{array}{r}
\bar{M}^{2} \simeq\left(M_{D} M_{D}^{\dagger}+H^{2}\right) \equiv M^{2} \\
\bar{m}^{2} \simeq K^{\dagger} m_{\text {eff }} m_{\text {eff }}^{\dagger} K
\end{array}
$$

with

$$
m_{e f f} m_{e f f}^{\dagger} \simeq M_{d} M_{d}^{\dagger}-\frac{\left(M_{d} M_{D}^{\dagger} M_{D} M_{d}^{\dagger}\right)}{M^{2}}
$$

Note that $K$ is the mixing matrix connecting standard quarks and has small deviations from unitarity given by $K^{\dagger} K=1-S^{\dagger} S$, with:

$$
S \simeq-\frac{M_{D} M_{d}^{\dagger} K}{M^{2}}\left(1+\frac{\bar{m}^{2}}{M^{2}}\right)
$$

It is the fact that second term in Eq. (8) may be of a magnitude similar to that of $M_{d} M_{d}^{\dagger}$ that makes it possible to rescue the four texture ansatz discussed before.

The present experimental data can be well reproduced [70], 64] from the following Froggatt-Nielsen pattern [71] for $m_{\text {eff }}$ :

$$
m_{e f f} \sim m_{b}\left(\begin{array}{ccc}
0 & \bar{\varepsilon}^{3} & \bar{\varepsilon}^{4} \\
\bar{\varepsilon}^{3} & \bar{\varepsilon}^{2} & \bar{\varepsilon}^{2} \\
\bar{\varepsilon}^{4} & \bar{\varepsilon}^{2} & 1
\end{array}\right)
$$

with $\bar{\varepsilon} \simeq 0.2$ together with a similar pattern for the up sector. However due to the different hierarchies of the quark mass matrices the $V_{C K M}$ matrix is specially sensitive to the down sector and we can still reproduce the present experimental data with the form chosen for $M_{u}$ in Eq. (1). 
The specific patterns in terms of $\bar{\varepsilon}$ chosen for $\mathcal{M}_{d}$ in order to render viable this Yukawa texture are discussed in our original work [40] where a numerical example can also be found.

We would like to stress the important fact that a sizeable effect in $m_{\text {eff }} m_{\text {eff }}^{\dagger}$ can be obtained even in the limit of an extremely heavy vectorial quark. For $M_{D} M_{D}^{\dagger}$ and $H^{2}$ of the same order of magnitude Eqs. (8) and (9) show that the desired effect can be obtained in $m_{\text {eff }} m_{\text {eff }}^{\dagger}$ and at the same time, deviations from unitarity of the $3 \times 3 V_{C K M}$ matrix are suppressed due to the smallness of the entries in $S$.

\section{Acknowledgments}

The author thanks the organizers of the CTP 2007 Symposiun on Sypersymmetry at LHC for the warm welcome at the Centre for Theoretical Physics at The British University in Egypt and the stimulating scientifical environment provided. This work was partially supported by Fundação para a Ciência e a Tecnologia (FCT, Portugal) through the projects POCTI/FNU/44409/2002, PDCT/FP/63914/2005, PDCT/FP/63912/2005 and CFTP-FCT UNIT 777 wich are partially funded through POCTI (FEDER).

\section{References}

[1] R. N. Mohapatra et al., arXiv:hep-ph/0510213 and references therein.

[2] M. B. Gavela, P. Hernandez, J. Orloff, O. Pene and C. Quimbay, Nucl. Phys. B 430, 382 (1994) arXiv:hep-ph/9406289].

[3] M. Fukugita and T. Yanagida, Phys. Lett. B 174 (1986) 45.

[4] B. Aubert et al. [BABAR Collaboration], Phys. Rev. Lett. 94, 161803 (2005) arXiv:hep-ex/0408127.

[5] R. Itoh et al. [Belle Collaboration], Phys. Rev. Lett. 95, 091601 (2005) arXiv:hep-ex/0504030].

[6] K. Abe et al. [Belle Collaboration], arXiv:hep-ex/0507037.

[7] G. Sciolla [BABAR Collaboration], Nucl. Phys. Proc. Suppl. 156, 16 (2006) arXiv:hep-ex/0509022. 
[8] A. Poluektov et al. [Belle Collaboration], Phys. Rev. D 70, 072003 (2004) arXiv:hep-ex/0406067).

[9] K. Abe et al. [BELLE Collaboration], Phys. Lett. B 624, 11 (2005) arXiv:hep-ex/0408106.

[10] K. Abe et al. [Belle Collaboration], arXiv:hep-ex/0411049.

[11] B. Aubert et al. [BABAR Collaboration], Phys. Rev. D 73, 112004 (2006) arXiv:hep-ex/0604037.

[12] A. Poluektov et al. [Belle Collaboration], Phys. Rev. D 73, 112009 (2006) arXiv:hep-ex/0604054.

[13] B. Aubert et al. [BABAR Collaboration], Phys. Rev. Lett. 93, 231801 (2004) arXiv:hep-ex/0404029].

[14] B. Aubert et al. [BABAR Collaboration], Phys. Rev. Lett. 95, 041805 (2005) arXiv:hep-ex/0503049.

[15] A. Somov et al., Phys. Rev. Lett. 96, 171801 (2006) arXiv:hep-ex/0601024.

[16] B. Aubert et al. [BABAR Collaboration], arXiv:hep-ex/0607098.

[17] V. M. Abazov et al. [D0 Collaboration], Phys. Rev. Lett. 97, 021802 (2006) arXiv:hep-ex/0603029].

[18] A. Abulencia et al. [CDF - Run II Collaboration], Phys. Rev. Lett. 97, 062003 (2006) [AIP Conf. Proc. 870, 116 (2006)] arXiv:hep-ex/0606027.

[19] F. J. Botella, G. C. Branco, M. Nebot and M. N. Rebelo, Nucl. Phys. B 725, 155 (2005) arXiv:hep-ph/0502133.

[20] Z. Ligeti, Int. J. Mod. Phys. A 20, 5105 (2005) |arXiv:hep-ph/0408267].

[21] L. Silvestrini, Int. J. Mod. Phys. A 21, 1738 (2006) arXiv:hep-ph/0510077.

[22] M. Bona et al., AIP Conf. Proc. 881, 210 (2007). 
[23] J. Charles et al. [CKMfitter Group], Eur. Phys. J. C 41, 1 (2005) arXiv:hep-ph/0406184. / / updated results and plots available at: http://ckmfitter.in2p3.fr

[24] F. J. Botella, G. C. Branco and M. Nebot, Nucl. Phys. B 768, 1 (2007) arXiv:hep-ph/0608100.

[25] E. Lunghi and A. Soni, arXiv:0707.0212 [hep-ph].

[26] B. Aubert et al. [BABAR Collaboration], Phys. Rev. Lett. 98, 211802 (2007) arXiv:hep-ex/0703020.

[27] M. Staric et al. [Belle Collaboration], Phys. Rev. Lett. 98, 211803 (2007) arXiv:hep-ex/0703036].

[28] Y. Nir, JHEP 0705, 102 (2007) arXiv:hep-ph/0703235.

[29] G. C. Branco, P. A. Parada and M. N. Rebelo, Phys. Rev. D 52, 4217 (1995) arXiv:hep-ph/9501347|.

[30] P. Ball, arXiv:0704.0786 [hep-ph].

[31] E. Golowich, J. Hewett, S. Pakvasa and A. A. Petrov, arXiv:0705.3650 [hep-ph].

[32] W. M. Yao et al. [Particle Data Group], J. Phys. G 33, 1 (2006).

[33] M. Misiak et al., Phys. Rev. Lett. 98, 022002 (2007) arXiv:hep-ph/0609232.

[34] M. Misiak and M. Steinhauser, Nucl. Phys. B 764, 62 (2007) arXiv:hep-ph/0609241.

[35] U. Haisch, arXiv:0706.2056 [hep-ph].

[36] S. Chen et al. [CLEO Collaboration], Phys. Rev. Lett. 87, 251807 (2001) arXiv:hep-ex/0108032].

[37] P. Koppenburg et al. [Belle Collaboration], Phys. Rev. Lett. 93, 061803 (2004) arXiv:hep-ex/0403004.

[38] B. Aubert et al. [BaBar Collaboration], Phys. Rev. Lett. 97, 171803 (2006) arXiv:hep-ex/0607071. 
[39] E. Barberio et al. [Heavy Flavor Averaging Group (HFAG) Collaboration], arXiv:0704.3575 [hep-ex].

and online update available at http://www.slac.stanford.edu/xorg/hfag/.

[40] G. C. Branco, M. N. Rebelo and J. I. Silva-Marcos, arXiv:hep-ph/0612252, to appear in Phys. Rev. D.

[41] P. Minkowski, Phys. Lett. B 67, 421 (1977).

[42] T. Yanagida, In Proceedings of the Workshop on the Baryon Number of the Universe and Unified Theories, Tsukuba, Japan, 13-14 Feb 1979

[43] S. L. Glashow, In "Quarks and Leptons", Cargèse, ed. M. Lévy et al Plenum, 1980 New York, p. $70 \%$.

[44] M. Gell-Mann, P. Ramond and R. Slansky, In Supergravity : proceedings, Stony Brook, N.Y., Sep. 27-29, 1979, ed P. Van Nieuwenhuizen and D.Z. Freedman. North-Holland 1979

[45] R. N. Mohapatra and G. Senjanovic, Phys. Rev. Lett. 44, 912 (1980).

[46] G. C. Branco, P. A. Parada and M. N. Rebelo, arXiv:hep-ph/0307119.

[47] Y. Achiman, Phys. Lett. B 599, 75 (2004) arXiv:hep-ph/0403309.

[48] B. Adhikary, J. Phys. G 32, 23 (2006) arXiv:hep-ph/0502245.

[49] G. 't Hooft, Phys. Rev. Lett. 37, 8 (1976).

[50] G. 't Hooft, Phys. Rev. D 14, 3432 (1976) [Erratum-ibid. D 18, 2199 (1978)].

[51] A. E. Nelson, Phys. Lett. B 136, 387 (1984).

[52] A. E. Nelson, Phys. Lett. B 143, 165 (1984).

[53] S. M. Barr, Phys. Rev. Lett. 53, 329 (1984).

[54] L. Bento, G. C. Branco and P. A. Parada, Phys. Lett. B 267, 95 (1991).

[55] G. C. Branco and L. Lavoura, Nucl. Phys. B 278, 738 (1986).

[56] Y. Nir and D. J. Silverman, Phys. Rev. D 42, 1477 (1990). 
[57] G. C. Branco, T. Morozumi, P. A. Parada and M. N. Rebelo, Phys. Rev. D 48, 1167 (1993).

[58] G. Barenboim, F. J. Botella, G. C. Branco and O. Vives, Phys. Lett. B 422, 277 (1998) arXiv:hep-ph/9709369].

[59] G. Barenboim, F. J. Botella and O. Vives, Nucl. Phys. B 613, 285 (2001) arXiv:hep-ph/0105306.

[60] D. Hawkins and D. Silverman, Phys. Rev. D 66, 016008 (2002) arXiv:hep-ph/0205011.

[61] J. A. Aguilar-Saavedra, Phys. Rev. D 67, 035003 (2003) [Erratum-ibid. D 69, 099901 (2004)] [arXiv:hep-ph/0210112.

[62] G. C. Branco, D. Emmanuel-Costa and R. Gonzalez Felipe, Phys. Lett. B 477 (2000) 147 arXiv:hep-ph/9911418.

[63] H. Fritzsch and Z. z. Xing, Prog. Part. Nucl. Phys. 45, 1 (2000) arXiv:hep-ph/9912358].

[64] R. G. Roberts, A. Romanino, G. G. Ross and L. Velasco-Sevilla, Nucl. Phys. B 615, 358 (2001) arXiv:hep-ph/0104088.

[65] L. J. Hall and A. Rasin, Phys. Lett. B 315 (1993) 164 arXiv:hep-ph/9303303.

[66] P. Ramond, R. G. Roberts and G. G. Ross, Nucl. Phys. B 406 (1993) 19 arXiv:hep-ph/9303320].

[67] R. Barbieri, L. J. Hall and A. Romanino, Phys. Lett. B 401 (1997) 47 arXiv:hep-ph/9702315.

[68] G. C. Branco, L. Lavoura and F. Mota, Phys. Rev. D 39 (1989) 3443.

[69] G. C. Branco, M. N. Rebelo and J. I. Silva-Marcos, Phys. Lett. B 597 (2004) 155 [arXiv:hep-ph/0403016].

[70] G. C. Branco, D. Emmanuel-Costa and R. Gonzalez Felipe, Phys. Lett. B 483 (2000) 87 arXiv:hep-ph/9905290.

[71] C. D. Froggatt and H. B. Nielsen, Nucl. Phys. B 147 (1979) 277. 